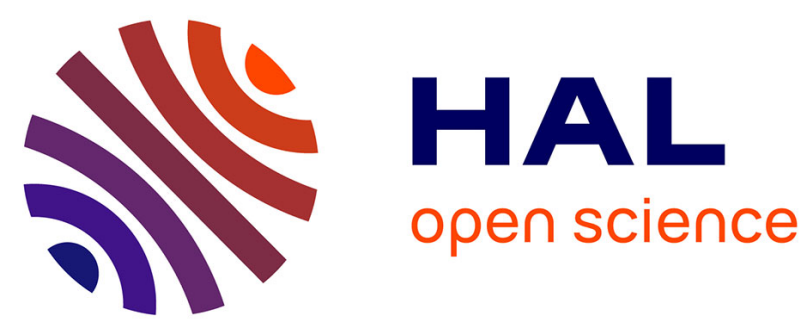

\title{
Comparative Analysis of Different Types of Sources on the Performance of Rigid Noise Barriers on Rigid Ground Using Analytical Formulae, a 2.5-D BEM Method and Scale Modelling Tests
}

Qiutong Li, Denis Duhamel, Honore Yin, Yanyun Luo

\section{To cite this version:}

Qiutong Li, Denis Duhamel, Honore Yin, Yanyun Luo. Comparative Analysis of Different Types of Sources on the Performance of Rigid Noise Barriers on Rigid Ground Using Analytical Formulae, a 2.5-D BEM Method and Scale Modelling Tests. Acta Acustica united with Acustica, 2019, pp.987-999. 10.3813/AAA.919379 . hal-02892324

\section{HAL Id: hal-02892324 \\ https://hal-enpc.archives-ouvertes.fr/hal-02892324}

Submitted on 7 Jul 2020

HAL is a multi-disciplinary open access archive for the deposit and dissemination of scientific research documents, whether they are published or not. The documents may come from teaching and research institutions in France or abroad, or from public or private research centers.
L'archive ouverte pluridisciplinaire HAL, est destinée au dépôt et à la diffusion de documents scientifiques de niveau recherche, publiés ou non, émanant des établissements d'enseignement et de recherche français ou étrangers, des laboratoires publics ou privés. 


\title{
Comparative analysis of different types of sources on the performance of rigid noise barriers on rigid ground using analytical formulae, a 2.5-D BEM method and scale modelling tests
}

\author{
Qiutong $\mathrm{LI}^{1), 2)}$, Denis Duhamel ${ }^{1)}$, Honore $\mathrm{YIN}^{1)}$ Yanyun LUO ${ }^{2)}$ \\ 1) Université Paris-Est, Laboratoire Navier, ENPC-IFSTTAR-CNRS, UMR 8205, \\ Ecole des Ponts ParisTech, France \\ 2) Institute of Rail Transit, Tongji University, Shanghai 201804, P.R. China.
}

\section{Summary}

With the aim of a perfect source model to simulate railway traffic noise within a shorter computational time, this paper compares coherent line, incoherent point and incoherent line sources on the performance of barriers by using an analytical solution, a 2.5-D BEM method and scale modelling tests. The comparison between the analytical solutions and the $2.5-\mathrm{D}$ BEM prediction results shows that the BEM calculations for a coherent line source can be used to approximately show the barrier attenuation spectrum for a one-point source and the single-number rating for an incoherent line source when the barrier is straight on the ground. Then, validations with scale modelling tests were performed outdoors under controlled conditions. The results obtained by using several loudspeakers radiating incoherent sounds simultaneously show good agreement with the 2.5-D BEM prediction results for the one-point source and incoherent point sources, not only for a simple barrier on the ground but also for a double-straight barrier on a viaduct. Based on these agreements, the frequency and longitudinal distance dependences on the barrier attenuation for incoherent point sources are discussed to understand the barrier attenuation spectrum for the incoherent line source.

\section{Introduction}

Noise barriers are widely used in traffic systems to reduce exposure to traffic noise in surrounding residential and commercial areas. The railway noise source is typically assumed to be an incoherent line source, but to predict the barrier performance within an acceptable computational time, a coherent line source (2-D BEM) or a one-point source facing the receiver (3-D BEM) is always considered as the alternative in the numerical calculations. Compared with the computational cost of 2-D BEM calculations, the cost for 3 -D calculations significantly increases due to the sophisticated matrix computations. Furthermore, be- cause the element size must be less than one-sixth the sound wavelength, the cost for higher frequency calculations is considerably high, even in two dimensions. The calculation time also depends on other parameters, such as the frequency range of interest and the absorptive surface treatments. The long calculation time is the main problem for solving 3-D BEM models, especially for barriers with complicated tops, and the calculations are often conducted using a 2-D BEM approach $[1,2,3,4]$ (with coherent line sources). In the early years, D.C. Hothersall et al.[1] discussed the 2-D BEM model of T-profile and associated noise barriers based on the results obtained from experimental modelling and field measurements. They found that the predicted results were not applicable to the incoherent line source, but the relative performances of different barrier shapes would be similar. I. Takashi et al.[2] studied the performance of road traffic noise barriers with various shapes and surface conditions using only a 2-D BEM method. When studying the efficiency of low-height noise reduction devices applied on the roadside, M. Baulac et al.[3] carried out 1:10 scale model measurements to confirm the effectiveness. They found good agreement between the 2D theoretical results and 3D scale model measurements. Moreover, F. Koussa et al.[4] studied the acoustic performance of conventional and low-height gabion noise barriers using a 2-D BEM model and scale model measurements. The agreement of the results between the two methods was satisfactory.

However, using 2-D BEM models for researching railway/road traffic noise was found to be inappropriate because the results obtained for these cases were noticeably different. P. Jean et al.[5] emphasised the importance of source type on the assessment of noise barriers. Using the Fourier-like transformation proposed by [6], they found that the barrier attenuation was overestimated if coherent line sources were considered, whereas the efficiency of a cap on the top of a straight barrier was underestimated with coherent line sources. Later, with the help of a BEM program that they compiled, their team[7] obtained the real 
performance of a T-shaped absorbing cap with road traffic noise conditions on the ground. They found that the results of cap efficiency for a coherent line source were different from those for an incoherent line source. For the highest frequencies, the efficiency was proportional to the path difference. They also found the slantwise propagation effects on the barrier attenuation for a point source when the source-receiver distance was not perpendicular to the barrier with a simple analytical formula. However, to date, there has been little research that can clarify the slantwise effects of the distance between the source and receiver along the barrier $\left|z_{s}-z_{r}\right|$ (in the third direction perpendicular to the cross-section plane, it will be given as "longitudinal distance" for clarity) on the performance of barriers with arbitrary shapes.

To reduce the computational time in 3-D BEM calculations, D. Duhamel[6, 8] proposed a 2.5-D method in which the results obtained for coherent line sources can be transformed via Fourier-like transformations to those corresponding to incoherent point or line sources. Using this method, many articles have predicted the performance of acoustic screens for incoherent point (or line) sources in different applications. Forssen et al.[9] compared the results predicted by a 2.5-D BEM method and the results obtained from an in situ measurement, which showed reasonable agreement. S. Sakamoto et al.[10] and M. Hiroe et al.[11] employed a Fourier-like transformation in a 2-D finitedifference time-domain analysis to study the noise shielding effect of eaves/louvres attached on building façades and the propagation of sound from surface railways. The calculation method was validated by the experimental results. Based on the above successful experiences, the present paper continues to use this 2.5-D method to compare the results of different types of sources to predict the performance of urban railway noise barriers.

The predictions must be validated by the measurement results obtained from outdoor in situ[9, 7] or scaled laboratory tests $[3,10,11,4]$. In situ measurements may be time-consuming, and it is difficult to find a real environment as simple as the numerical model(rigid barrier, rigid flat ground, no reflecting obstacles, etc.), even if background noise can be rejected using controlled signals(such as ESS, MLS) and the intrinsic characteristics of noise barriers can be measured in situ with a given reproducibility[12]. However, performing large-scale measurements requires extremely large anechoic laboratories that are not easy to build and run. Hence, only a few studies $[13,14,15,16]$ related to in situ measurements have been published.

Because of the difficulties in conducting in situ measurements discussed above, a measurement method in which small-scale model tests are used instead offers a reliable alternative for predicting performance. Many articles[17, 18, 19] have used the scale modelling method to understand the propagation of $\mathrm{road} / \mathrm{railway}$ traffic noise to the surrounding environment, and the scaled measurement method has been widely employed in the study of noise barrier performance[20, 21, 22, 23, 24, 25]. Based on the invariance of the speed of sound in air, the performance of real barriers in the field can be imitated by the results of scale models, which is possible when the measured frequency range is increased by the same scale factor to the typical range of interest for the urban railway traffic noise. The scaled approach is perfectly suited for our research because we focus on comparing different source types to evaluate the performance of a simple barrier on the ground and a double-straight barrier on a viaduct, which are assumed to be rigid throughout. In addition, it is known that the impedance of surfaces must be scaled with complicated computations, not as that of an acoustic rigid surface, which is infinite. Such surfaces with absorbent treatments are not considered in this paper.

Various sound sources, such as air-jet and electroacoustic sources, laser-generated acoustic pulses and electric sparks, have been used during the measurement process, depending on the scale-modelling application. G.R. Watt et al.[20] used an air-jet whistle activated by an air supply at 10 atmospheres to simulate an omni-directional point source. Among the different source types that are able to provide these characteristics, spark discharge in air is an interesting solution. Many studies have presented the characteristics of the spark discharge, which can be regarded as an adjustable acoustic source for scale model measurements $[19,26,23,3]$. For researching the propagation of explosions and sonic booms conveniently in the laboratory, Q. Qin et al.[27] investigated the characteristics of acoustical shock waves associated with a focused pulsed laser beam. Aiming at modelling incoherent point sources, our approach is to use scaled outdoor experiments and several point sound sources. The sound radiated simultaneously by several miniature loudspeakers with uncorrelated white noises is easily considered to be that of incoherent point sources. This approach can validate the prediction results not only for the one-point source but also for the incoherent point sources, thus providing a new avenue for predicting the results for an incoherent line source.

The main purpose of the present work is to determine whether the assumption of coherent line sources is acceptable for predicting the performance of rigid noise barriers on rigid ground. Section 2 preliminarily examines the effects of different source types on a simple straight barrier on the rigid ground, with three configurations of source and receiver positions, using a 2.5-D BEM method and an analytical solution. This analysis can provide a preliminary explanation for the comparison of different source types. In Section 3, a scale model technique is developed with 
the help of miniature loudspeakers, and a set of scaled measurements is presented with a short description of the set-up; the results and comparisons between the measured and predicted results are then discussed. Section 4 is devoted to the frequency dependence and longitudinal distance dependence of the barrier attenuation for the incoherent point sources, which better characterises the barrier performance in the case of incoherent point or line sources. Some conclusions are then presented in Section 5.

\section{Comparison with an analyti- cal solution}

In this section, our objective is to seek a much closer approximation to the real solution for the sound field due to an incoherent line source in the vicinity of a sound barrier. For simplicity, the time-dependent factor of $e^{-\mathrm{i} w t}$ is understood and omitted from the whole computation process. Suppose that the distance between the source and receiver is $R$, and therefore, the acoustic field for a free space is $e^{\mathrm{i} k R} / 4 \pi R$ assuming the customary source term of $-\delta\left(x-x_{s}\right)$ [28]. In [28], K.M. Li sorted many different analytical models for calculating the sound diffraction by a thin infinite barrier. Among these models, we selected one of the frequently used exact solutions, which was developed by MacDonald[29], for comparison with the results predicted by the BEM approach for a one-point source. The expression of the sound field in the shadow zone was recast by Bowman and Senior[30] in the cylindrical polar system due to the original idea solved using the spherical polar coordinate, given as follows:

$$
\begin{aligned}
p_{D}= & \frac{\mathrm{i} k \operatorname{sgn}\left(\zeta_{1}\right)}{4 \pi} \int_{\left|\zeta_{1}\right|}^{\infty} \frac{H_{1}^{(1)}\left(k R_{1}+s^{2}\right)}{\sqrt{s^{2}+2 k R_{1}}} d s \\
& +\frac{\mathrm{i} k \operatorname{sgn}\left(\zeta_{2}\right)}{4 \pi} \int_{\left|\zeta_{2}\right|}^{\infty} \frac{H_{1}^{(1)}\left(k R_{2}+s^{2}\right)}{\sqrt{s^{2}+2 k R_{2}}} d s
\end{aligned}
$$

where $\mathrm{i}$ is the imaginary number, $k$ is the wave number of the incident wave, and $H_{1}^{(1)}$ is the Hankel function of the first kind. $\zeta_{1}$ and $\zeta_{2}$ are the limits of the contour integrals, which are determined by

$$
\zeta_{1,2}=\operatorname{sgn}\left(\left|\theta_{s}-\theta_{r}\right|-\pi\right) \sqrt{k\left(R^{\prime}-R_{1,2}\right)}
$$

where $R_{1}$ and $R_{2}$ are determined by

$$
R_{1,2}=\sqrt{r_{s}^{2}+r_{r}^{2}-2 r_{s} r_{r} \cos \left(\theta_{s} \mp \theta_{r}\right)+\left(y_{s}-y_{r}\right)^{2}}(3)
$$

Moreover, the shortest source-edge-receiver path can be determined by

$$
R^{\prime}=\sqrt{\left(r_{s}+r_{r}\right)^{2}+\left(y_{s}-y_{r}\right)^{2}}
$$

where $\left(r_{s}, \theta_{s}, y_{s}\right)$ and $\left(r_{r}, \theta_{r}, y_{r}\right)$ are the cylindrical coordinates of the source and receiver, respectively.
Note the lack of consideration of the sound reflection induced by the ground because the solution was deduced starting from the assumption of a semi-infinite screen. Generally, sound reflection by the rigid ground or a rigid viaduct is tacitly included in the performance of a sound barrier for road/rail traffic systems. Based on the pertinent theory, the sound reflecting from the surface of the rigid ground or a rigid viaduct can be considered to radiate in terms of an image source located symmetrically with the infinite plane. Likewise, the effect on the receiver side can be described as an image receiver. Consequently, the total sound field influenced by the barrier's diffraction together with the ground's reflection is the summation of four diffracted paths when the surface is fully reflective. This symmetrical method is introduced in the post process of the calculation to allow the solution to approximate that for the case where the noise barrier is on the rigid ground in outdoor situations but is not exactly the same because the barrier is semi-infinite in the analysis, whereas it is of finite height in reality.

In the present study, using a 2.5-D BEM approach to model the sound field generated from coherent line and incoherent point and line sources, the existing program SAMRAY developed by Duhamel $[6,8]$ was introduced. The solution for a coherent line source is generally known as a 2-D BEM result that can easily be calculated. Then, via a Fourier-type integration, the solution for a one-point source can possibly be obtained from a series of 2-D results when all the boundaries are assumed to be acoustically rigid[6]. The calculations for incoherent line sources can also be made by 2-D solutions, which have been discussed in [6]. Note that the solution for an incoherent line source is represented by the density of acoustic potential energy because the source is modelled as a line of uncorrelated point sources perpendicular to the cross-section of the barrier. Based on these discussions, the existing program allows calculating the radiation and the diffraction of sound fields for general 2-D and 2.5-D structures for coherent line, point and incoherent line sources. Considering the totally reflective ground effect, the comparison of predictions calculated by SAMRAY with the analytical solution is described here for three cases:

1. A source and a receiver located on the totally reflective ground.

2. A source located on the ground, and a receiver $1.0 \mathrm{~m}$ above the totally reflective ground.

3. A source $1.0 \mathrm{~m}$ above the ground, and a receiver $1.5 \mathrm{~m}$ above the totally reflective ground.

Figure 1 shows diagrams of the three configurations and the 2-D coordinates of the source and the receiver. The straight barrier was assumed to be totally reflective as well, with a height of $1.85 \mathrm{~m}$ and a thickness of $0.17 \mathrm{~m}$. To compare the analytical solutions for a 
one-point source with the BEM results for a coherent line source, a one-point source and an incoherent line source, the barrier attenuation $A t t_{b}$, which is the sound pressure difference between the site without a barrier and the site with a barrier was introduced and is given by

$$
\operatorname{Att}_{b}(f)=10 \log \frac{p_{\mathrm{wo}}^{2}(f)}{p_{\mathrm{w}}^{2}(f)}
$$

where $p_{\mathrm{wo}}(f)$ and $p_{\mathrm{w}}(f)$ denote the sound pressure at the given receiver position in the case of the models without and with a barrier, respectively. Figure 2 shows the barrier attenuation as a function of frequency calculated by each analytical or numerical model for each case. Each model was run at $0.1 \mathrm{~Hz}$ from $44.7 \mathrm{~Hz}$ to $112 \mathrm{~Hz}$ and at $1 \mathrm{~Hz}$ from $113 \mathrm{~Hz}$ to $5623 \mathrm{~Hz}$. In Figure 2, each dotted curve represents the barrier attenuations in the frequency spectrum whereas the solid curve of the same colour corresponds to the results in one-third-octave bands from $50 \mathrm{~Hz}$ to $5000 \mathrm{~Hz}$. Thus, the latter appears to be much smoother than the former. In case 1 (Figure $1(\mathrm{a}))$, both the source and the receiver are located on the ground such that there is no need to consider the reflection effect in terms of the ground. Without the ground effect, Figure 2(a) only shows the component diffracted by the barrier top. As shown in the figure, the increase in the barrier attenuation is proportional to the rise in the logarithm of frequency, regardless of the source type. However, in case 2, considering the ground effect on the side of the receiver, the barrier attenuation varies regularly with frequency for the coherent line source and the one-point source. The period of the variation depends on the path difference between the direct way of sound transmission and the reflecting way, which is governed by the height of the receiver above the ground. Furthermore, as shown in Figure 2(c), with the source and the receiver at different heights above the ground, the barrier attenuation varies irregularly like a combination of two different periodic variations.

Compared with the results for the coherent line source and the one-point source, those for the incoherent line source (yellow curves) are distinctly different. The sound pressure levels for the incoherent line source were calculated by the energy density for an infinite line of incoherent point sources such that the corresponding barrier attenuation at each frequency could be considered as the averaged results for the uncorrelated point sources with different longitudinal distances. Section 4.2 will elaborate the effect of the longitudinal distance on the attenuation of barriers. Hence, the trend for the incoherent line source shown in Figure 2 is in accordance with the average characteristic that the barrier attenuation increases with frequency much more slowly than the other results. For this reason, the curve of the one-third-octave spectrum can be used to more precisely describe the fre-

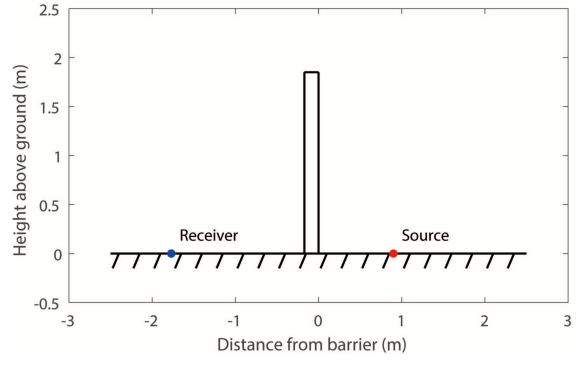

(a) Case 1

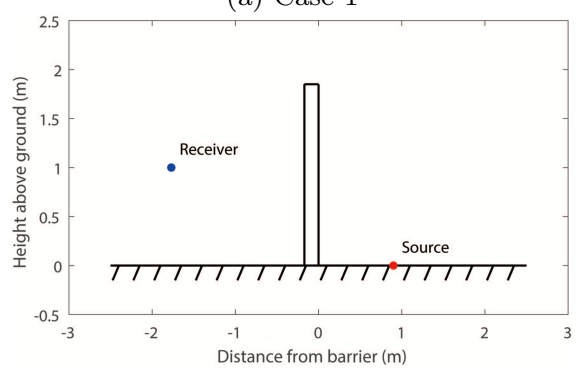

(b) Case 2

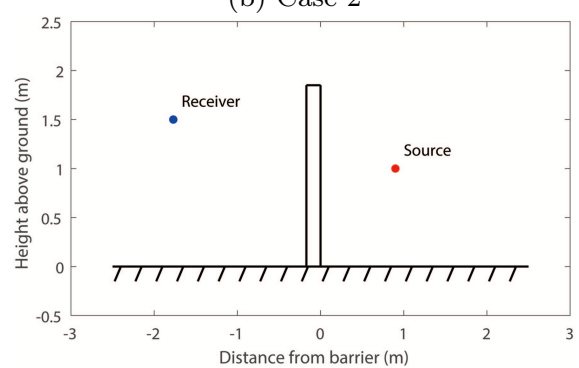

(c) Case 3

Figure 1: Cross-sections of the three configurations calculated in the comparison with the analytical solution

quency spectrum for the incoherent line source. When considering incoherent line sources in further studies, the estimation results calculated for central frequencies of one-third octave bands are sufficient to show an accurate spectrum. Another conclusion in [6] can be observed in Figure 2: the barrier attenuations for the coherent line source in each subfigure have good agreement with that for the one-point source in the spectrum, while in the one-third-octave band analysis, the barrier attenuations for the one-point source are slightly higher than those for the coherent line source. Because the result for the one-point source is generated by the Fourier-like transformation of that for the coherent line source, it is not surprising to obtain this conclusion. For this reason, when considering a onepoint source in further studies, the calculations for a coherent line source can serve as the alternative to reduce the computational time. In addition, it is also indicated that the totally reflective ground effect had little influence on the comparison of these sources.

The red curves presented in Figure 2 represent the analytical solutions for the one-point source used to validate the numerical predictions calculated by SAMRAY. Clearly, the analytical solutions have good 


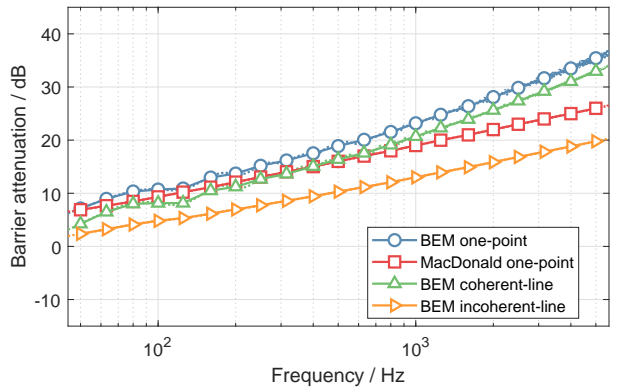

(a) Case 1

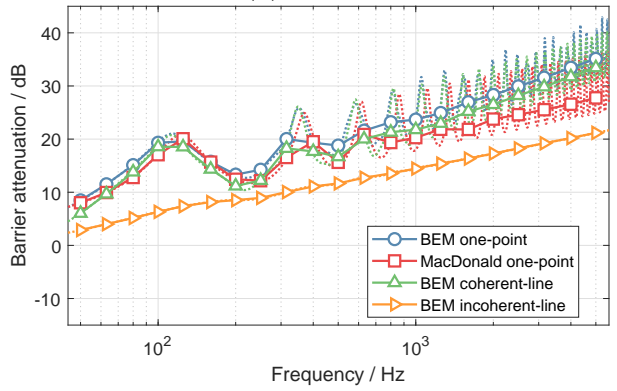

(b) Case 2

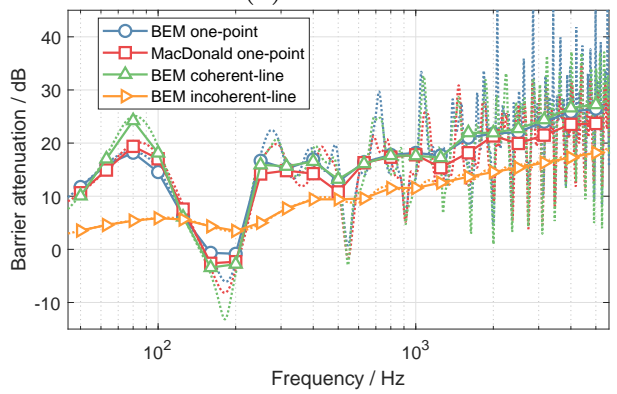

(c) Case 3

Figure 2: Barrier attenuation spectra for the three configurations calculated in the comparison

agreement with those predicted by the BEM at low frequencies. However, at mid and high frequencies, the analytical solution becomes much lower, and the variation period becomes much longer. The reason for this result lies in the assumption of the barrier thickness. At the beginning of the analytical calculation, the barrier was assumed to be thin with a thickness of zero. However, in the calculation process of the 2.5D BEM modelling, the thickness could be modelled equivalently to that of the actual barrier. Figure 3 compares the analytical solution for case 1 with the 2.5-D BEM results predicted for the barrier with different thicknesses. It is clear that the differences between the analytical solution and the BEM results are small at low frequencies, free from the change in thickness. However, with an increase in frequency, the difference is considerably increasing, which is caused by the increased thickness. Thus, it was validated that the results predicted by SAMRAY must be closer to the actual values due to the consideration of the barrier thickness, particularly for the results at mid and high frequencies.

Except for the frequency spectrum, we al-

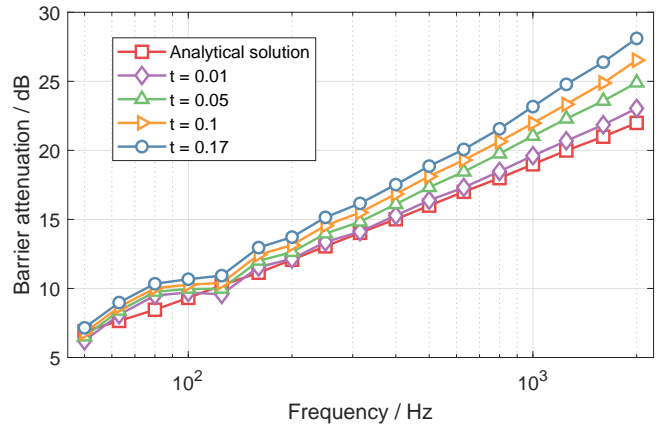

Figure 3: The analytical solution for case 1 compared with the 2.5-D BEM results of the barrier with different thicknesses (units: $\mathrm{m}$ )

ways use a single-number rating within the frequency range of interest to analyze the barrier performance. The single-number rating is often called "insertion loss" (IL), which can be given by

$$
\mathrm{IL}=10 \log \frac{\int_{f_{\min }}^{f_{\max }} p_{\mathrm{wo}}^{2}(f) d f}{\int_{f_{\min }}^{f_{\max }} p_{\mathrm{w}}^{2}(f) d f}
$$

where $f_{\min }$ and $f_{\max }$ are the lower and upper limits of the frequency range, respectively.

According to the norm ISO 10847:1997[31], the frequency range for the railway traffic noise is recommended to range from $50 \mathrm{~Hz}$ to $5000 \mathrm{~Hz}$. To compare the single-number ratings for the one-point source between the two calculation methods, we find from Table 1 that the results predicted by the 2.5-D BEM were 2-3 dB higher than those obtained from the analytical solutions, which were the results of the assumed thin barriers in the analytical model. Notably, by comparing the insertion losses predicted by the 2.5 D BEM program for different source types, the results for the coherent line source are in good agreement with those for the incoherent line source but are much lower than those predicted for the one-point source. This result is why many studies considered the sound field radiated by a coherent line source as that for an incoherent line source, although the results observed in the frequency spectrum are completely contrary to each other. Hence, it is indicated that the insertion loss for the coherent line source can be used to estimate the value for the incoherent line source to reduce the computational time.

\section{Comparison with scale mod- elling tests}

With the assumption of the actual thicknesses of barriers, the 2.5-D BEM prediction results must be much closer to the actual values compared with the analytical solutions. However, the predictions for the three 
Table 1: Insertion losses for three configurations for different types of sources (frequency range: 50-5000 $\mathrm{Hz}$ )

\begin{tabular}{ccccc}
\hline \multirow{2}{*}{ IL / dB } & $\begin{array}{c}\text { Analytical } \\
\text { solution }\end{array}$ & \multicolumn{3}{c}{ Predicted results by BEM } \\
\cline { 2 - 5 } & One-point & One-point & $\begin{array}{c}\text { Coherent } \\
\text { line }\end{array}$ & $\begin{array}{c}\text { Incoherent } \\
\text { line }\end{array}$ \\
\hline Case 1 & 19.7 & 22.9 & 11.7 & 14.2 \\
Case 2 & 21.3 & 24.1 & 14.8 & 15.2 \\
Case 3 & 17.7 & 19.7 & 13.4 & 13.0 \\
\hline
\end{tabular}

cases need to be validated using the scale modelling method. In addition to the three cases for a one-point source discussed in Section 2, a case with a doublestraight barrier installed on a box girder viaduct(case 4) was introduced to more realistically study the railway noise barrier system. Nevertheless, it remains quite difficult to model the incoherent line source that is commonly used to more closely reflect the traffic noise. Because the incoherent line source can be considered an infinite line of uncorrelated point sources perpendicular to the cross-section, several incoherent point sources were introduced into the test and performed for cases 3 and 4 . Consequently, the scale model measurement with several point sources was made to not only validate the 2.5 -D BEM prediction results for the one-point source but also for the incoherent point sources. Due to the size limitation of the experiment site, the scales of cases 1-3 were determined to be 1:10, whereas that of case 4 was 1:20.

\section{$3.1 \quad$ Test setup}

The tests for the one-point source were performed in the four abovementioned cases. For the first three, the solid plane barrier remained unchanged at 18.5 $\mathrm{cm}$ high and $1.7 \mathrm{~cm}$ wide. The site was an open field on unknown asphalt. To ensure good acoustic reflection to simulate rigid surface conditions, a wood plank with an area of $1.2 \times 1.8 \mathrm{~m}^{2}$ was placed on the asphalt (as shown in Figure 4), which was sufficiently large to offer an approximate rigid ground in our scale model test. Figure 4 also shows that a layer of sand was inserted to fill the air gap between the plank and the asphalt to eliminate the influence of vibration of the panel and the air cavity resonance effect under the plank surface.

For case 4 , the barriers and the viaducts were made of 9 -mm-thick wood panels. The scale model was based on a real prototype and is shown in Figure $5(\mathrm{a})$, with a length of $6 \mathrm{~m}$. Double-straight barriers with a height of $2.4 \mathrm{~cm}$ were located on the box girder viaduct, which was $50 \mathrm{~cm}$ above the ground supported by discontinuous piers, with the gap between each two being $1 \mathrm{~m}$. The receiver was positioned towards the centre of the model where there was no pier. Figure

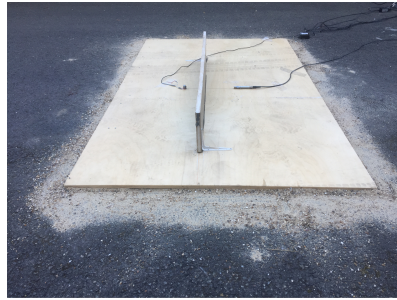

(a) Case 1, one-point source

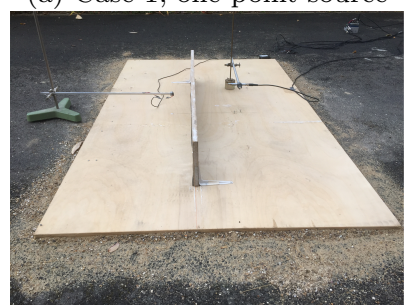

(c) Case 3, one-point source(d) Case 3, incoherent point sources

Figure 4: The scenes of the scale model tests for the former three cases

5 (b) shows the cross-section of the real model. Due to the large vehicle structure, secondary reflections pose a problem, and thus, the train itself had to be taken into account in the scale modelling tests. The T-shape part in the centre of the viaduct was designed as a safe passage. Since the viaduct was the closest reflective surface to the source and was elevated above the ground, the acoustical characteristic of the ground seemed a lot less important. Hence, there was no need to place the wood plank on the asphalt in case 4 .

To evaluate the performance of the barriers, it was necessary to prepare the configurations without barriers. For the first three cases only, the straight barrier was removed, and for case 4 , the double-straight barrier was removed(as shown in Figure 5(c)). The positions of the loudspeakers and microphones were unchanged. To describe the positions of the sources and receivers for each case, the horizontal distance to the surface of the barrier was denoted by $x$, the vertical distance to the ground was denoted by $y$, and the longitudinal distance to the microphone along the barrier was denoted by $z$. Figures 1 and 5 show the coordinates for each real model while Table 2 illustrates the coordinates for both the real and the scale models. The tests for a one-point source, where the perpendicular from the source to the receiver meets the $\operatorname{barrier}\left(z_{r}=z_{s}=0\right)$, were performed first. Then, the tests for incoherent point sources were conducted with the number of sources increased for cases 3 and 4 , with other coordinates of loudspeakers and microphones remaining constant. Note that in each case, the height of the receiver was less than that of the barrier, which is a result of the need to keep the receiver well within the shadow zone.

The BEM model assumes omni-directional incoherent point sources, which were achieved in practice by using miniature loudspeakers (produced by RS PRO, RS stock code: 1176047), activated by am- 
Table 2: Positions of the loudspeakers and microphones in three coordinates $(\mathrm{m})$

(a) For a one-point source

\begin{tabular}{|c|c|c|c|c|c|c|c|c|c|}
\hline \multirow{2}{*}{ Real model } & \multicolumn{2}{|c|}{ Microphone } & \multicolumn{2}{|c|}{ Loudspeaker } & \multirow{2}{*}{ Scale model } & \multicolumn{2}{|c|}{ Microphone } & \multicolumn{2}{|c|}{ Loudspeaker } \\
\hline & $x_{r}$ & $y_{r}$ & $x_{r}$ & $y_{r}$ & & $x_{r}$ & $y_{r}$ & $x_{r}$ & $y_{r}$ \\
\hline Case 1 & 1.6 & 0.0 & 0.9 & 0.0 & Case 1 & 0.16 & 0.00 & 0.09 & 0.00 \\
\hline Case 2 & 1.6 & 1.0 & 0.9 & 1.0 & Case 2 & 0.16 & 0.10 & 0.09 & 0.10 \\
\hline Case 3 & 1.6 & 1.5 & 0.9 & 1.0 & Case 3 & 0.16 & 0.15 & 0.09 & 0.10 \\
\hline Case 4 & 2.0 & 16.1 & 2.42 & 12.4 & Case 4 & 0.10 & 0.805 & 0.121 & 0.62 \\
\hline
\end{tabular}

(b) For incoherent point sources

\begin{tabular}{|c|c|c|c|c|c|c|c|c|c|c|c|c|c|}
\hline \multirow{2}{*}{ Real model } & \multicolumn{13}{|c|}{ Loudspeaker } \\
\hline & Num & & & & & & $z_{s}$ & & & & & & \\
\hline \multirow{3}{*}{ Case 3} & 1 & & & & & & & 0.00 & & & & & \\
\hline & 3 & & & & & & -0.35 & 0.00 & 0.35 & & & & \\
\hline & 12 & -2.10 & -1.75 & -1.40 & -1.05 & -0.70 & -0.35 & 0.00 & 0.35 & 0.70 & 1.05 & 1.40 & 1.75 \\
\hline \multirow{3}{*}{ Case 4} & 1 & & & & & & & 0.00 & & & & & \\
\hline & 4 & & & & & -19.78 & -7.18 & 0.00 & 12.60 & & & & \\
\hline & 12 & -59.34 & 46.74 & -39.56 & -26.96 & -19.78 & -7.18 & 0.00 & 12.60 & 19.78 & 32.38 & 39.56 & 52.16 \\
\hline \multirow{2}{*}{ Scale model } & \multicolumn{13}{|c|}{ Loudspeaker } \\
\hline & Num & \multicolumn{12}{|c|}{$z_{s}$} \\
\hline \multirow{3}{*}{ Case 3} & 1 & & & & & & & 0.0 & & & & & \\
\hline & 3 & & & & & & -3.5 & 0.0 & 3.5 & & & & \\
\hline & 12 & -21.0 & -17.5 & -14.0 & -10.5 & -7.0 & -3.5 & 0.0 & 3.5 & 7.0 & 10.5 & 14.0 & 17.5 \\
\hline \multirow{3}{*}{ Case 4} & 1 & & & & & & & 0.0 & & & & & \\
\hline & 4 & & & & & -98.9 & -35.9 & 0.0 & 63.0 & & & & \\
\hline & 12 & -296.7 & -233.7 & -197.8 & -134.8 & -98.9 & -35.9 & 0.0 & 63.0 & 98.9 & 161.9 & 197.8 & 260.8 \\
\hline
\end{tabular}

plifiers (Viston, AMP 2.2 LN, Art. No. 7102) and a power supply (EA-PS 2042-10B). The sound radiated from the speakers was generated by a signal output module (NI 9263) installed in a NI DAQ system (CDAQ-9174). The effective maximum frequency of the miniature loudspeaker was up to $20 \mathrm{kHz}$ in the one-third octave band. Together with the frequency range of railway traffic noise recommended in ISO 10847:1997(50-5000 Hz), the measured frequency ranges in cases 1-3 were determined to be $500 \mathrm{~Hz}$ to $20 \mathrm{kHz}$, and that in case 4 was $1000 \mathrm{~Hz}$ to $20 \mathrm{kHz}$. Hence, the measured results can simulate a 50-2000 $\mathrm{Hz}$ emission for cases 1-3 and a 50-1000 Hz emission for case 4 in the real-size problem.

During the measurement, one or more loudspeakers simultaneously emitted random white noise in the same one-third octave band for 10 seconds from the signal output module. Meanwhile, sound pressure signals were received by microphones and transferred to the signal input module. The ten-second random white noise was based on continuous integrated sound pressure levels, so the barrier end effects had to be limited. To limit the end effects, the receiver was positioned towards the centre of the barrier, and both barrier ends were filled with mineral wool to absorb the sound diffracted by the ends. Each test was repeated five times.

All the tests were conducted in the same place. The tests of each case with and without the barrier were carried successively at the site, lasting for approximately one hour in total. In the duration of the test for each configuration, the effect of humidity and temperature on air absorption of high frequencies was considered unchanged. Since the attenuation of the straight or double-straight barrier that was of our interest was the difference in level between the sites with and without barriers, the effect of humidity and temperature could be ignored. Nevertheless, the temperature during the tests was measured, as presented in Table 3.

Table 3: Temperature of $\operatorname{tests}\left({ }^{\circ} \mathrm{C}\right)$

\begin{tabular}{cccc}
\hline & Number of & \multicolumn{2}{c}{ Configurations } \\
& loudspeakers & Without a barrier & With a barrier \\
\hline Case 1 & 1 & 22.1 & 21.8 \\
Case 2 & 1 & 21.9 & 22.0 \\
& 1 & 22.3 & 22.2 \\
Case 3 & 3 & 22.2 & 22.2 \\
& 12 & 22.3 & 22.5 \\
& 1 & 17.8 & 17.6 \\
Case 4 & 4 & 17.7 & 17.5 \\
& 12 & 17.8 & 17.9 \\
\hline
\end{tabular}




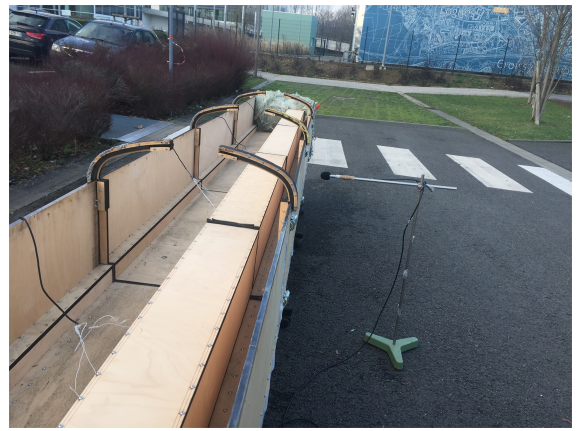

(a) The scene of the scale model

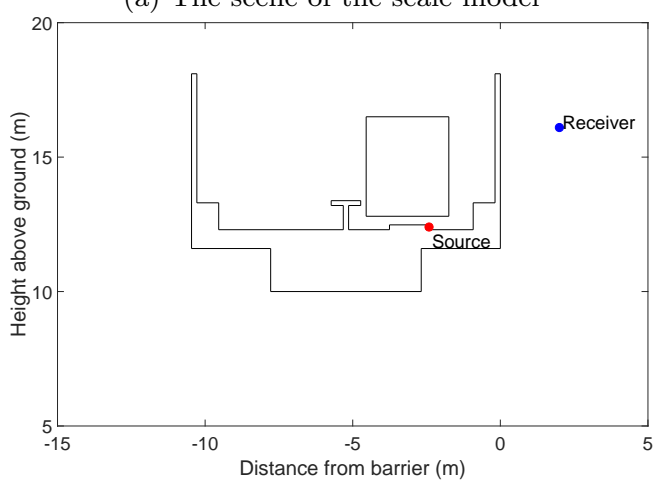

(b) The cross-section with a barrier in the BEM calculation

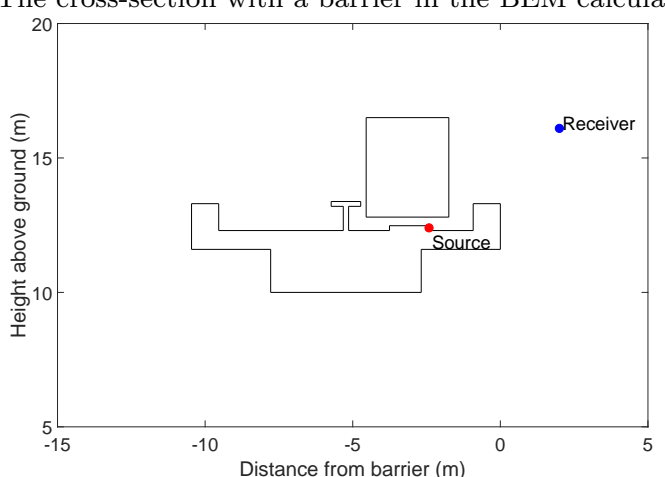

(c) The cross-section without a barrier in the BEM calculation

Figure 5: Configurations of case 4

\section{$3.2 \quad$ Test results compared to the $2.5-\mathrm{D}$ BEM predictions}

Predictions were performed for the straight and double-straight barriers using the 2.5-D BEM program SAMRAY. The one-point source in the model was placed in exactly the same position as for the scale model tests. The number of sources defined was initially one for modelling the one-point source, followed by adding sources to reach three or four and finally reaching twelve sources. The barrier attenuation of the one-point source for each case was calculated by using Eq (5). By contrast, to yield the results by the combined effect of different incoherent point sources, the barrier attenuation for incoherent point sources was given by

$$
A t t_{b, \text { sum }}(f)=10 \log \frac{\sum_{i=1}^{N} p_{\text {wo }}^{2}\left(f, z_{s i}\right)}{\sum_{i=1}^{N} p_{\mathrm{w}}^{2}\left(f, z_{s i}\right)}
$$

where $p_{\mathrm{wo}}\left(f, z_{s i}\right)$ and $p_{\mathrm{w}}\left(f, z_{s i}\right)$ denote the sound pressure at the given receiver position radiated from the source located at $z_{s i}$ in the case of the models without and with the barrier, respectively. $N$ denotes the number of incoherent point sources. The barrier attenuations at one-third-octave band frequencies from $50 \mathrm{~Hz}$ to $2000 \mathrm{~Hz}$ were calculated to be compared for the first three tested cases, whereas those from 50 $\mathrm{Hz}$ to $1000 \mathrm{~Hz}$ were calculated for case 4 .

For the one-point source that was perpendicular to the receiver, Figure 6 shows plots of the measured and predicted barrier attenuations by the one-third-octave band spectrum for all four cases. The frequency range of the measured spectrum was adjusted in the analysis to be identical to the predicted results. Hence, the frequencies will be given in full scale for clarity. As expected, there are good agreements between the measured results obtained in the scale model tests and those predicted by the 2.5-D BEM approach. However, the measured barrier attenuations are slightly higher than those predicted by the BEM, particularly for high frequencies. This result was considered to be normal and permissible due to the sound absorption of the wood panels and the non-idealised point source in the scale test.

In Figure 7, the results for different numbers of

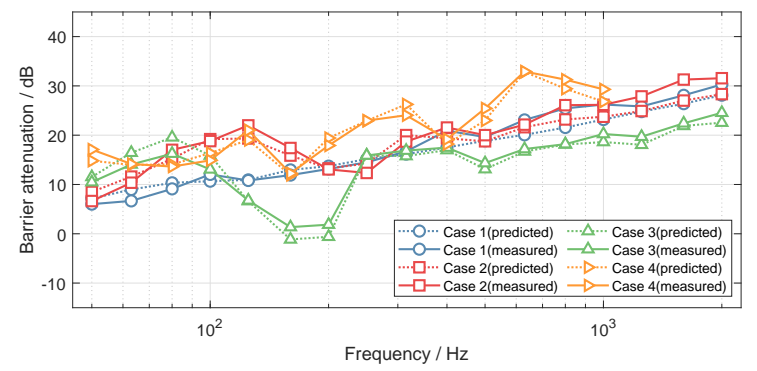

Figure 6: Measured and predicted barrier attenuations for the one-point source

loudspeakers in cases 3 and 4 are compared. Here, the simultaneous sound sources were lined up along the length of the barrier, only differing by the longitudinal distance. As shown in Figure 7, the measured result for each case in general has a good agreement with the prediction, which indicates that by using the 2.5-D BEM approach, the predicted barrier attenuations for incoherent point sources are accurate as well. Looking into the details, there are discrepancies at the peaks $(80 \mathrm{~Hz}$ and $200 \mathrm{~Hz})$ for case 3 , which can be caused by the warping tendency of the wood plank on the ground. It is also clear that all the curves 


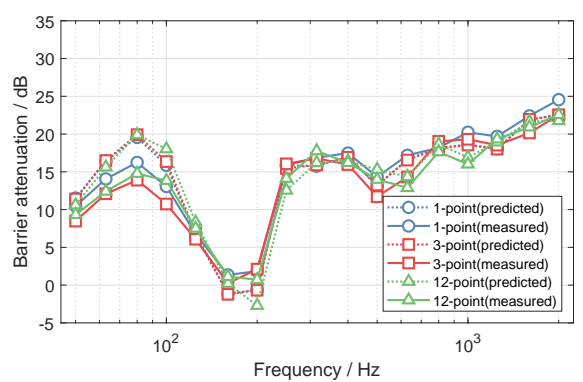

(a) Case 3

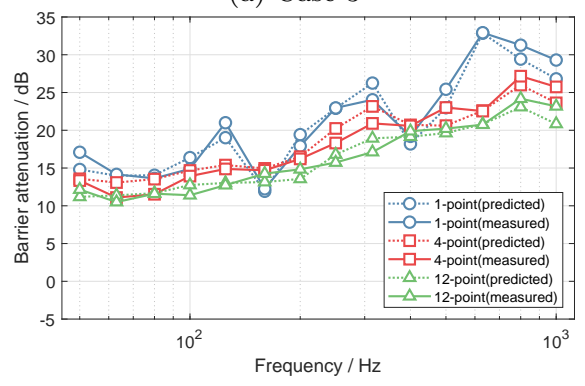

(b) Case 4

Figure 7: Measured and predicted barrier attenuations for incoherent point sources

in Figure 7(a) are too close to distinguish from each other. This result means that the number of incoherent point sources has little effect on the attenuation of the straight barrier on the ground. Nevertheless, there is no proof that the number effect can be ignored when referring to the barrier attenuation for incohert point sources.

For the double-straight barrier on the viaduct, as . Fin in $7(\mathrm{~b})$, it is easy to understand that growth of the barrier attenuation seriously flucto gradually increase as the number of incoherent point sources increased to four. When the number of sources increased to twelve, the barrier attenuation has a slight decrease at each frequency band compared with those for four-point sources.

\section{Discussion}

The attenuation of a barrier varies with the sound frequency. Moreover, the barrier attenuation also changes with the number of incoherent point sources because the longitudinal distances for incoherent point sources are diverse. Therefore, the attenuation of a barrier can be affected by the longitudinal distance. Based on the two cases from which the results were validated by the scale model tests, this section will discuss the frequency dependence and the longitudinal distance dependence of the barrier attenuation.

\subsection{Frequency dependence}

The frequency dependence for case 3 was discussed in section 2. The barrier attenuation varies as a combination of two different periodic variations in the frequency spectrum, and the period is mainly dependent on the path difference between the direction way and the reflection way governed by the height of the source and the receiver above the ground. For case 4 , Figure 8 shows the predictions of the barrier attenuations in the spectrum of 50-2000 Hz. Unlike the results for the simple straight barrier on the totally reflective ground, the barrier attenuation for the onepoint source violently fluctuates regardless of sound frequency, as does that for the coherent line source. As discussed in [32], a reflecting barrier fixed on the source side could result in the deterioration of barrier performance due to the acoustic resonance effect. Hence, the resonance effect can be a reasonable explanation of the valleys illustrated in Figure 8. Nevertheless, there are indistinct valleys at the resonant frequencies in the configuration with four-point and twelve-point sources and even no visible valleys in the configuration with the incoherent line source. Considering that the resonance effect did not disappear upon changing the longitudinal distance, these smoother trends must have a relationship with the calculated barrier attenuation for incoherent point sources.

From Eq (7), it appears that the barrier attenuation, the ratio of the whole sound power of the model without and with the barrier, is the average of the results for all the uncorrelated point sources. Then, our focus is shifted to the frequency spectrum for each incoherent point source. Figure 8(c) shows the one-third-octave spectrum for part of twelve point sources in case 4 , where $\left|z_{s i}-z_{r}\right|$ denotes the longitudinal distance for the $i$ th point source. As shown, with a change in the longitudinal distance, the barrier attenuation spectrum performed significantly differently along the sound frequency. When the whole sound power for such calculated point sources was obtained, the averaged characteristics must result in the decrease of peaks and valleys in the spectrum. The decreasing trend is more evident as the number of sources or the longitudinal distance increases. Assuming that the incoherent line source is a line of such incoherent point sources with an extremely small distance between each two sources along the line that is infinitely long perpendicular to the cross-section, the averaged characteristics lead to the smoothest barrier attenuation, as shown by the purple curve in Figure 8(b). However, for the coherent line source, there is almost no change in the frequency spectrum in terms of the different longitudinal distances in the assumption, and therefore, the frequency spectrum corresponding to the coherent line source performed almost the same as that for the one-point source. In Figure 8(b), there is good agreement for the one-third-octave spectrum 


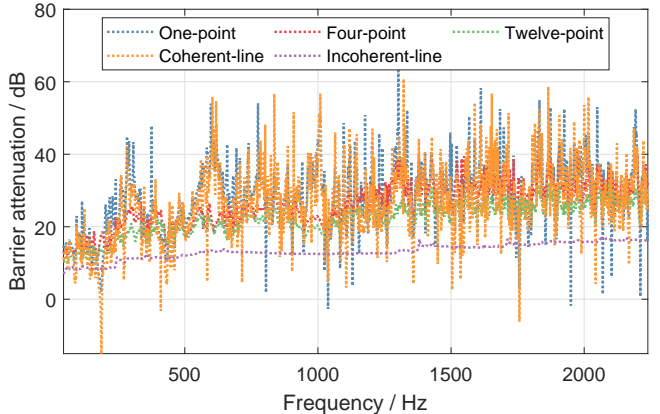

(a) Frequency spectra

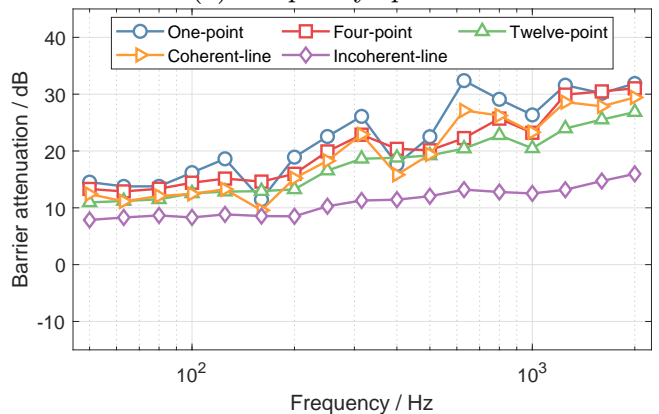

(b) $1 / 3$ octave band spectra

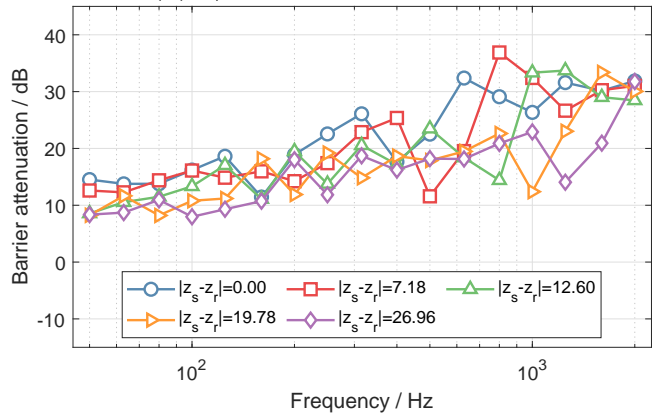

(c) Spectra for each one-point source with different longitudinal distances

Figure 8: The spectra of barrier attenuations for case 4 calculated by the 2.5 -D BEM approach

between the one-point source and the coherent line source, which provides further evidence that the calculation in the frequency domain for a coherent line source can be considered an alternative to analyse the barrier performance with a one-point source. In addition, the spectrum for the coherent line source is slightly lower than that for a one-point source, which is due to the averaged characteristics of the coherent line source.

The single-number ratings for different numbers of incoherent point sources are illustrated in Table 4 . With different numbers of incoherent point sources, the insertion loss for case 3 is essentially unchanged. By contrast, the insertion loss in case 4 decreases considerably with the number of sources and becomes closer to that for the incoherent line source, which is identical to the comparison in the frequency spectrum. The different trends for cases 3 and 4 could be due to the longitudinal distance dependence, which will be detailed in Section 4.2. Another comparison of the insertion loss is between the one-point source and the coherent line source. The former is higher than the latter at approximately $6 \mathrm{~dB}$ for both cases, although their frequency spectra were almost the same, as mentioned above. Then, the single ratings for the coherent line source and the incoherent line source manifest similarly, with the former being higher than the latter by only $2 \mathrm{~dB}$, embodying the "line" intrinsic feature of the assumption of the coherent line source. In conclusion, the results for a coherent line source have presented not only a frequency spectrum that is approximate to that for the one-point source but also a single-number rating that is approximate to that for the incoherent line source.

\subsection{Longitudinal distance dependence}

As explained above, the change in longitudinal distance of the point source results in significant differences in the barrier attenuation spectrum. Because the incoherent line source was considered a line of such point sources, according to Eq (7), the average of such a spectrum for each distance can be the resulting spectrum for the incoherent line source, which is notable because of its smoothness and slow growth with frequency. Hence, studying the longitudinal distance dependence is of great importance. To clarify the relationship between the longitudinal distance of the point source and the barrier attenuation, the spectra of the barrier attenuation in both cases 3 and 4 for each incoherent point source with different longitudinal distances were calculated. Figure 9 shows the predicted barrier attenuation spectra with filled contours when the longitudinal distance is within 100 meters. The $\mathrm{x}$ axis denotes the longitudinal distance, and the $y$ axis denotes the sound frequency on a log scale. For comparison, the contours for both cases 3 and 4 use the same colour map.

In Figure 9(a), increasing the longitudinal distance generally results in the shift of the spectrum to higher frequencies, approximately following a linear relationship. Then, the spectrum for every nonzero longitudinal distance is therefore divided into two components by frequencies: the relatively lower values at low frequencies and the spectrum for the previous distance at mid and high frequencies(e.g., for the longitudinal distance of $40 \mathrm{~m}$, the barrier attenuations at $200-2000 \mathrm{~Hz}$ have the same trend as those in the whole frequency range for $10 \mathrm{~m}$, whereas the barrier attenuations below $200 \mathrm{~Hz}$ are lower). In addition, there are closed contours periodically in the relationship between the spectrum and longitudinal distance(e.g., orange closed contours), which cannot be identified in the spectrum only for the one-point source or the coherent line source. With the help of the longitudinal distance dependence, the barrier attenuation for the incoherent line source can be presented thoroughly.

The filled contour in Figure 9(b) is excessively com- 
Table 4: Single ratings for different types of sources

\begin{tabular}{cccccc}
\hline IL / dB & \multicolumn{5}{c}{ Predicted results by the 2.5-D BEM } \\
\hline Case 3 & One-point & Three-point & Twelve-point & Coherent line & Incoherent line \\
$(50-5000 \mathrm{~Hz})$ & 19.7 & 19.9 & 19.8 & 13.4 & 13.0 \\
Case 4 & One-point & Four-point & Twelve-point & Coherent line & Incoherent line \\
$(50-2000 \mathrm{~Hz})$ & 20.5 & 19.4 & 17.3 & 13.7 & 11.8 \\
\hline
\end{tabular}

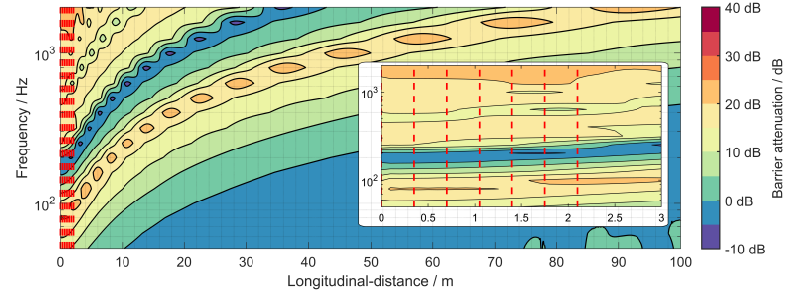

(a) Case 3, the straight barrier on the ground, source-receiver distance perpendicular to the barrier: $2.67 \mathrm{~m}$

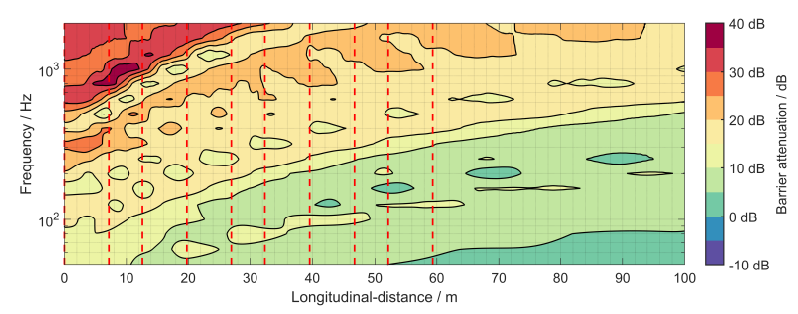

(b) Case 4, the double-straight barrier on the viaduct, source-receiver distance perpendicular to the barrier: $4.415 \mathrm{~m}$

Figure 9: The relationships between the longitudinal distance and the barrier attenuation spectrum

plicated mainly due to the acoustic resonance effect caused by the double-straight barrier, generally with much higher levels than those in Figure 9(a). At first glance, the contour of $35 \mathrm{~dB}$ occurs at frequencies from $800 \mathrm{~Hz}$ to $1250 \mathrm{~Hz}$ at distances of $5 \mathrm{~m}$ to $12 \mathrm{~m}$ but not at a distance of zero, which is another finding highlighting the importance of the longitudinal distance for modelling incoherent point or line sources. Secondly, identical to Figure 9(a), the spectrum shifts to higher frequencies with increasing longitudinal distance. However, the relationship cannot be approximate to the linearity, and at some frequencies, it can be described with logarithmic curves. The spectrum for the longer distance decreases at low frequencies and remains at high levels at mid and high frequencies. At a distance of $100 \mathrm{~m}$, the spectrum still appears higher than $10 \mathrm{~dB}$ at frequencies above $500 \mathrm{~Hz}$, which means that even the longitudinal distance is approximately twenty-five times the source-receiver distance perpendicular to the barrier and that the sound pressure level at the receiver position is still greatly affected by the barrier attenuation. In view of this result, when considering modelling of the incoherent line source, the frequency spectrum for the coherent line or one-point source cannot be an acceptable alternative.
In general, the longitudinal distance dependence for a specific case must be analysed specifically due to the significant difference between these two contours.

To explain the different effects of the number of the incoherent point sources on the barrier attenuation results between cases 3 and 4, the barrier attenuation spectra for those incoherent point sources tested in the scale experiments are marked by red dashed lines, as shown in Figure 9. Because the red lines are densely packed together for case 3 , the part including all the red lines is zoomed in and shown by a small picture in Figure 9 (a). It can be seen that the barrier attenuation spectra in case 3 for each tested point source are almost the same because the distances between each point source along the barrier in case 3 are too short to cover the entire area of the longitudinal distance dependence. For this reason, the calculations for case 3 must result in the identity of the results for one-point, three-point and twelve-point sources, which is in accordance with the experimental results shown in Figure 8(a) and Table 4. However, in case 4, the more complex relationship between the distance and the barrier attenuation spectrum causes the results for each point source to be more diverse than those in other studies. Consequently, the results for each tested point source are considerably different, as marked by red dashed lines shown in Figure 8(b). As a result, as the number of incoherent point sources increases, the spectrum for case 4 clearly changes. In conclusion, the study on the longitudinal distance dependence of barrier attenuation through 2.5-D BEM modelling provides a reasonable explanation for the experimental results.

\section{Conclusion}

In this paper, the attenuations of a rigid straight barrier on the rigid ground and a double-straight barrier on a rigid viaduct generated from different types of sources have been investigated. A first comparison has been achieved by the analytical solution proposed by MacDonald and the 2.5-D BEM predictions by SAMRAY, able to obtain the similarities and differences among the one-point source, coherent line source and incoherent line source. Then, a measurement procedure using several loudspeakers radiating incoherent sounds simultaneously with two scale models has been presented to verify the 2.5-D BEM calculations for different numbers of incoherent point 
sources. From the 2.5-D BEM results, it has been possible to determine the frequency and longitudinal distance dependence of the barrier attenuation spectrum for incoherent point sources to introduce it into modelling the spectrum for an incoherent line source. In addition, the single-number rating for the frequency range of interest has also been analysed for all configurations.

The following conclusions can be drawn from the predictions and measurements:

1. The solutions of the analytical formulae show good agreement with the 2.5-D BEM calculations (for a one-point source) both in the spectrum and the single-number rating. This result validates the 2.5-D BEM calculation program, but to calculate the barrier attenuation for a onepoint source, it is better to use the BEM approach rather than the analytical formulae due to the concretization of the barrier thickness. With increasing simple barrier thickness, the barrier attenuation increases more obviously at higher frequencies.

2. As expected, there is good agreement of the barrier attenuation spectrum at a given receiver position between a one-point source (2.5-D BEM) and the corresponding coherent line source (2D BEM). Hence, from the perspective of saving time, the 2-D BEM method can be directly used to estimate the attenuation spectrum of the barrier for a one-point source. However, the singlenumber rating for a coherent line source (2-D BEM) seriously underestimates that for a onepoint source, the former being lower than the latter by $6-10 \mathrm{~dB}$, which cannot be directly used for the estimation of a one-point source.

3. With the characteristics of "line", the singlenumber rating results for a coherent line source are close to the calculations for an incoherent line source (within an error of $3 \mathrm{~dB}$ ), although the barrier attenuation spectra between them are noticeably different. Hence, the single-number rating for a coherent line source (2-D BEM) can be used to estimate that for an incoherent line source.

4. By using several loudspeakers radiating incoherent sounds simultaneously, the scale modelling test results show good agreements with the 2.5D BEM calculations for both the configurations of the straight barrier on the ground and the double-straight barrier on a viaduct. The presented scale modelling test method can be used for the certification of incoherent point sources in a laboratory and in situ.

5. The presented scale modelling test results validate the 2.5-D BEM calculations for incoherent point sources of a simple model, as well as a complex model that is typical of urban railway transit configurations. Hence, the BEM approach generalised to predict the attenuation of rigid barriers on rigid ground for an incoherent line source can be reliable, even for a more sophisticated rigid barrier model on rigid ground.

6. The results of the double-straight barrier obtained for a coherent line source or a one-point source facing the receiver fluctuate violently, mainly depending on the acoustic resonance induced by the multiple reflections between the two parallel barriers.

7. An increased number of incoherent point sources can result in the barrier attenuation spectrum becoming smoother and lower and lead to a clear decrease in single-number ratings for the whole frequency range of interest. Nevertheless, the barrier attenuation still increases with frequency with a lower growth rate.

8. Using the 2.5-D BEM method, the sourcereceiver direction was introduced. Unlike the invariance under translation in the distance of the spectrum for a coherent line source, the spectrum for an incoherent point source shifts to higher frequencies with increasing longitudinal distance, and the barrier attenuations at low frequencies generally decrease with increasing longitudinal distance.

9. The smoother and lower barrier attenuation spectra for the incoherent line source can be explained by the dependence on the longitudinal distance of the incoherent point source. As the incoherent line source is assumed to be a line of incoherent point sources, the barrier attenuation spectrum for the incoherent line source can be solved by the ratio of the whole sound energy integral of incoherent point sources along the line for the model without and with a barrier. With the average characteristics, the spectrum becomes smoother and lower.

10. In this way, the testing of a new barrier employed on an urban railway transit can be performed by means of scale model measurements for incoherent point sources, as well as 2.5-D BEM calculations. However, the barrier performance in reality under the metro operating conditions should be obtained through 2.5-D BEM calculations for incoherent line sources. For a preliminary crude investigation, the single-number rating obtained for a coherent line source can be used to predict the real performance of the barrier. 


\section{Acknowledgement}

This work was supported in part by the National Natural Science Foundations of China(Nos. 51708422, $51678446,51408434)$. The authors would like to acknowledge many people who were involved in this work. Extra thanks go to Christophe BERNARD, Daniel CINTRA, Gwendal CUMUNEL, Zhehao ZHU and Yichun LIU for their additional valuable help. The authors also wish to thank the China Scholarship Council and Ecole Nationale des Ponts et Chaussees for providing the financial assistance to LI Qiutong necessary to pursue her Ph. D. in France.

\section{References}

[1] D. C. Hothersall, D. H. Crombie, and S. N. Chandler-Wilde. The performance of t-profile and associated noise barriers. Applied Acoustics, 32(4):269-287, 1991.

[2] Takashi Ishizuka and Kyoji Fujiwara. Performance of noise barriers with various edge shapes and acoustical conditions. Applied Acoustics, 65(2):125-141, 2004.

[3] Marine Baulac, Jérôme Defrance, Philippe Jean, Florence Minard, Marine Baulac, and Florence Minard. Efficiency of noise protections in urban areas: predictions and scale model measurements. Acta Acustica United with Acustica, 92(4):530-539, 2006.

[4] F. Koussa, J. Defrance, P. Jean, and P. BlancBenon. Acoustic performance of gabions noise barriers: Numerical and experimental approaches. Applied Acoustics, 74(1):189-197, 2013.

[5] P. Jean, J. Defrance, and Y. Gabillet. The importance of source type on the assessment of noise barriers. Journal of Sound and Vibration, 226(2):201-216, 1999.

[6] D. Duhamel. Efficient calculation of the threedimensional sound pressure field around a noise barrier. Journal of Sound and Vibration, 197(5):547-571, 1996.

[7] J. Defrance and P. Jean. Integration of the efficiency of noise barrier caps in a $3 \mathrm{D}$ ray tracing method. Case of a T-shaped diffracting device. Applied Acoustics, 64(8):765-780, 2003.

[8] D. Duhamel and P. Sergent. Sound propagation over noise barriers with absorbing ground. Journal of Sound and Vibration, 218(5):799-823, 1998.
[9] Jens Forssén, Laura Estévez-Mauriz, Clas Torehammar, and Philippe Jean. A low-height acoustic screen in a setting with an urban road: measured and predicted insertion loss. In Internoise, 2016.

[10] Shinichi Sakamoto and Ami Aoki. Numerical and experimental study on noise shielding effect of eaves/louvers attached on building façade. Building and Environment, 94:773-784, 2015.

[11] Masaaki Hiroe, Tomohiro Kobayashi, and 1003 Satoshi Ishikawa. 2.5-Dimensional finitedifference time-domain analysis for propagation of conventional railway noise: Application to propagation of sound from surface railway and its verification by scale model experiments. Acoustical Science and Technology, 38(1):42-45, 2017.

[12] M. Garai, E. Schoen, G. Behler, B. Bragado, M. Chudalla, M. Conter, J. Defrance, P. Demizieux, C. Glorieux, and P. Guidorzi. Repeatability and reproducibility of in situ measurements of sound reflection and airborne sound insulation index of noise barriers. Acta Acustica united with Acustica, 2014.

[13] Jeffrey Parnell, Stephen Samuels, and Con Tsitsos. The acoustic performance of novel noise barrier profiles measured at the roadside. Acoustics Australia, 38(3):123-128, 2010.

[14] Haibo Wang, Peng Luo, and Ming Cai. Calculation of noise barrier insertion loss based on varied vehicle frequencies. Applied Sciences, 8(1):100, 2018.

[15] M. Garai and P. Guidorzi. In situ measurements of the intrinsic characteristics of the acoustic barriers installed along a new high speed railway line. Noise Control Engineering Journal, 56(5):342-355, 2008.

[16] H. G. Jonasson. Sound reduction by barriers on the ground. Journal of Sound and Vibration, 22(1):113-126, 1972.

[17] H. W Jones, D. C Stredulinsky, and P. J Vermeulen. An experimental and theoretical study of the modelling of road traffic noise and its transmission in the urban environment. Applied Acoustics, 13(4):251-265, 1980.

[18] M. E. Delany, A. J. Rennie, and K. M. Collins. A scale model technique for investigating traffic noise propagation. Journal of Sound and Vibration, 56(3):325-340, 1978.

[19] J. Picaut and L. Simon. A scale model experiment for the study of sound propagation in urban areas. Applied Acoustics, 62(3):327-340, 2001. 
[20] G. R. Watts, D. C. Hothersall, and K. V. Horoshenkov. Measured and predicted acoustic performance of vertically louvred noise barriers. Applied Acoustics, 62(11):1287-1311, 2001.

[21] D. N. May and N. M. Osman. Highway noise barriers: new shapes. Journal of Sound and Vibration, 71(1):73-101, 1980.

[22] Tomonao Okubo and Kohei Yamamoto. Procedures for determining the acoustic efficiency of edge-modified noise barriers. Applied Acoustics, 68(7):797-819, 2007.

[23] Sergey I. Voropayev, Nicholas C. Ovenden, Harindra J. S. Fernando, and Paul R. Donovan. Finding optimal geometries for noise barrier tops using scaled experiments. The Journal of the Acoustical Society of America, 141(2):722-736, 2017.

[24] Mitsuyasu Yamashita and Kohei Yamamoto. Scale model experiments for the prediction of road traffic noise and the design of noise control facilities. Applied Acoustics, 31(1-3):185196, 1990.

[25] K. A. Mulholland. The prediction of traffic noise using a scale model. Applied Acoustics, 12(6):459-478, 1979.

[26] P. Bhuripanyo, S.I. Voropayev, and H.J.S. Fernando. Insertion loss spectrums behind straight noise barriers: Scaled experiments. In 2015 International Conference on Sustainable Energy and Environmental Engineering. Atlantis Press, 2015.

[27] Qin Qin and Keith Attenborough. Characteristics and application of laser-generated acoustic shock waves in air. Applied Acoustics, 65(4):325340, 2004.

[28] K.M. Li and H.Y. Wong. A review of commonly used analytical and empirical formulae for predicting sound diffracted by a thin screen. Applied Acoustics, 66(1):45-76, 2005.

[29] H. M. Macdonald. A class of diffraction problems. Proceedings of the London Mathematical Society, 14(1):410-427, 1915.

[30] J. J. Bowman, T. B. A. Senior, and P. L. E. Uslenghi. Electromagnetic and acoustic scattering by simple shapes (Revised edition). 1969.

[31] ISO/TC 43/SC 1 Noise. Acoustics - In-situ determination of insertion loss of outdoor noise barriers of all types, 1997.

[32] Yang Cheng, Pan Jie, and Cheng Li. A mechanism study of sound wave-trapping barriers. The Journal of the Acoustical Society of America, 134(3):1960-9, 2013. 